measurement uncertainty; it gives a general outline of what is involved and directs the reader to the document of interest. In addition, all other GUM documents focus on a specific purpose, for example:

- Application of the Gauss law of uncertainty propagation (JCGM 100:2008)

- Propagation of distributions (JCGM 101:2008)

- Measurements involving multiple measurands (JCGM 102:2011)

- Measurement models (JCGM 103)

- Least squares methods (JCGM 107)

- Bayesian methods (JCGM 108)

- Interlaboratory comparisons (JCGM 109)

This new structure emphasizes that users with different measurement problems require different guidance, which will now be available in stand-alone documents. For example, to apply the Monte Carlo method for a single measurand, the reader will only need JCGM 101:2008. The new structure enables the JCGM to respond in a flexible manner to emerging needs from the measurement community.

\section{JCGM 103}

Significant efforts at the JCGM-WG1 are spent in finalizing the document JCGM 103 which deals with developing and using measurement models. This document discusses the uncertainties that are inherent in the choice of models: theoretical, empirical, or hybrid. Uncertainties arise when analysts are faced with making choices between competing theoretical models (what approximations to make?), competing statistical models (fixed effects or random effects models), or competing empirical models (linear or quadratic approximation, what splines to adopt?). The JCGM 103 also discusses numerical issues that arise in nonlinear models and ways to deal with them (reparametrization, improved numerical methods, or change of mathematical framework in empirical models (monomial vs Chebyshev representation, for example)). The Committee draft will be circulated shortly.

\section{Other Business}

Two manuscripts of general interest have been published in Metrologia by members of the JCGM-WG1. The first document (Possolo and Pintar 2017 Metrologia 54 617; https://doi.org/10.1088/1681-7575/aa7e4a) discusses the plurality of choices that can reasonably be made when performing uncertainty evaluation. The manuscript shows that different choices typically lead to different estimates of the quantities of interest, and to different evaluations of the associated uncertainty.
This manuscript lends support for the new paradigm of the GUM whereby the various choices within the uncertainty estimation are welcomed by the equal status of the various GUM documents.

The second document (Cox and Shirono $2017 \mathrm{Me}$ trologia 54 642; https://doi.org/10.1088/1681-7575/ aa787f) addresses the 'small $n$ problem' which has been arguably the most critiqued aspect of the revised draft GUM of 2014. In short, non-informative Bayesian approach to uncertainty evaluation from a number $n$ of replicate observations provides standard uncertainty of the mean $(\mathrm{s} / \operatorname{sgrt}(n))^{*} \operatorname{sqrt}((n-1) /(n-$ $3)$ ) instead of the frequentist estimate of $s / \operatorname{sqrt}(n)$. The inapplicability of the former expression in situations when $n<4$ has drawn lots of criticism from IUPAC and IFCC, among others. This article provides informative Bayesian estimate of uncertainty applicable for all $n(n>1)$ based on prior knowledge for the upper bound of the standard uncertainty.

\section{Acknowledgments}

Portions of this summary were drawn from internal JCGM-WG1 document JCGM-WG1-N17- 13 drafted by Adriaan van der Veen (VSL, The Netherlands).

\section{For further information, contact Juris Meija <Juris.Meija@nrc-cnrc.gc.ca>, IUPAC representative on JCGM}

\section{DIGChem-a vision for chemical data standards}

DIGChem, the Data Interest Group/Chemistry, is an effort to foster a culture of data sharing within the chemistry community. In order to accomplish this vision, DIGChem is analyzing the existing landscape of chemistry data standards and chemical data repositories, evaluating and updating existing standards, analyzing the need for domain specific repositories, and advocating for educating researchers, librarians, publishers, and vendors on the benefits of research data sharing.

Anyone in the broader chemistry community who is interested is welcome to join the discussions. This is collaborative work between IUPAC Committee on Publications and Cheminformatics Data Standards (CPCDS), Subcommittee on Cheminformatics Data Standards (SCDS), the Research Data Alliance (RDA), Chemistry Research Data Interest Group (CRDIG), and the GO FAIR Chemistry Implementation Network (ChIN).

A number of working groups are being formed around areas of need and interest. Visit the following 
for linking to projects and sharing documentation: https://sites.google.com/view/digchem/

DIGChem was launched during the webinar presented by David Martinsen on 16 Feb 2018 and showcasing how SCDS is working across organizations to enable accurate exchange of chemical data within a FAIR data environment (FAIR = Findable, Accessible, Interoperable, Reusable). The presentation is available from the DIGChem website.

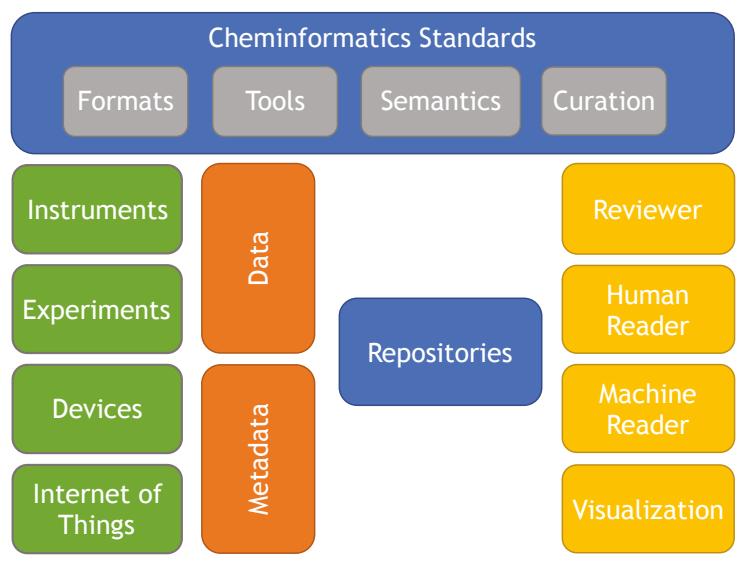

In conjunction with these working groups, a workshop is planned for 16-17 July 2018 in Amsterdam. This workshop, organized by IUPAC and CODATA (the Committee on Data for Science and Technology of the International Council for Science), will focus specifically on developing a GO FAIR network for chemistry, enabling access to chemical data by other domains, and spectral data standards and tools. For more details see https://iupac.org/event/supporting-fairexchange-chemical-data-standards-development/

For further information, contact Leah McEwen and Dave Martinsen at https://iupac.org/body/036.

\section{Collection, compilation and evaluation of elemental and} isotopic data of calcium carbonate and hydroxyapatite materials and the assessment of their usability to act as reference materials

Isotopic information of (calcium)carbonates and hydroxyapatites are widely used in different fields of research in order to study provenance, migration, authenticity, and past records of environmental conditions (e.g. in climate change adaption). The objective of this project is to collect and compile data on the isotopic composition and mass fractions (with a focus on $\mathrm{Sr}, \mathrm{Pb}, \mathrm{B}, \mathrm{Ba}, \mathrm{Li}, \mathrm{Mg}$ ) of materials based on calcium carbonates and hydroxy apatites.

This project will evaluate the data with respect to the metrological quality of the analytical approach. For the first time, the isotope user community will be provided with metrologically sound values relative to the accepted $\mathrm{O}$-anchor materials for isotopic delta data in materials. This is of significant importance especially for solid sample analysis (e.g. by Laser ablation multi collector inductively coupled plasma mass spectrometry (LA-MC ICP-MS)). The values compiled within the project will be based on internationally published data as well on laboratory results. International laboratories (with a focus on metrological institutes) will be contacted so that they may provide their according data. The evaluation of the data will be based on metrological principles and according to the best practices of the IUPAC Commission on Isotopic Abundances and Atomic Weights (CIAAW). Along with the data, uncertainties of measurements will be collected and evaluated. A combined standard uncertainty budget will be calculated and an uncertainty value will be assigned to the recommended isotope values for the corresponding materials. The dataset will be provided to the stakeholders via the CIAAW homepage as well as scientific publications.

For more information, contact the Task Group Chair Thomas Prohaska $<$ thomas.prohaska@boku.ac.at> https://iupac.org/project/2017-023-2-200

\section{Glossary of terms used in biochar research}

Biochar is a solid material obtained from the carbonization thermochemical conversion of biomass in an oxygen-limited environments. In more technical terms, biochar is produced by thermal decomposition of organic material (biomass such as wood, manure, leaves, husks) under limited supply of oxygen $\left(\mathrm{O}_{2}\right)$, and commonly at temperatures below $700^{\circ} \mathrm{C}$. When applied to soil, some biochars have been found to decrease nutrient leaching, enhance $\mathrm{pH}$, improve soil carbon concentrations and soil fertility. Additionally, biochars encourage moisture retention and aeration and have been shown to contribute towards reduced methane and other greenhouse gases emissions.

Biochar research is highly interdisciplinary; it is based on the use of analytical techniques (material characterization), and examination of its soil, water 